\title{
Three-Dimensional Dissipative Optical Solitons in a Dielectric Medium with Quantum Dots
}

\author{
M.Yu. Gubin ${ }^{1}$, A.Yu. Leksin ${ }^{1}$, S.M. Arakelian ${ }^{1}$, M.G. Gladush ${ }^{2}$, and A.V. Prokhorov ${ }^{1 a}$ \\ ${ }^{1}$ Vladimir State University, 87 Gorkogo Str., Vladimir 600000, Russia \\ ${ }^{2}$ Institute for Spectroscopy of RAS, 5 Fizicheskaya Str., Troitsk, Moscow 142190, Russia
}

\begin{abstract}
We consider the problem of formation of three-dimensional spatio-temporal dissipative solitons (laser bullets) in a dense ensemble of two-level quantum dots. The principal possibility of effective laser bullets generation in an all-dielectric metamaterials with quantum dots is shown. The phenomenon arises due to the simultaneous appearance of strong local field effects and significant corrections to diffraction effects during the propagation of short optical pulses in such medium.
\end{abstract}

We consider the problem of propagation optical pulse $\mathbf{E}_{\mathbf{p}}$ in an optically transparent all-dielectric metamaterial with two types of quantum dots. The quantum dots (type a) are assumed to be passive with small initial population of excited level, whereas the initial inversion of active quantum dots (type b) can be achieved by the action of a strong additional pump pulse. The main feature of the presented model is the accounting for both, i.e., dispersive and dissipation, local-field effects associated with the complex representation of the refractive index $n=n_{R}+i n_{I}$ for the host medium of the dielectric metamaterial. The contribution of these effects to the general picture of development of nonlinear processes is proportional to the Lorentz local-field parameter $\xi_{0}=\rho_{a}\left|\mu_{a}\right|^{2} /\left(3 \hbar \varepsilon_{0}\right)$, which can be large enough for quantum dots to provide new conditions for observation of self-induced transparency [1]. The system of equations for the density matrix elements (type a) in a host medium with a complex refractive index (permittivity) has the form:

$$
\begin{gathered}
\dot{\rho}_{11}=-i \Omega_{0}\left(\rho_{12}-\rho_{21}\right)+2 \xi_{0} u_{I}\left|\rho_{12}\right|^{2}+\Gamma_{\varepsilon} \rho_{22}, \\
\dot{\rho}_{12}=i\left(\Omega_{0}+\xi_{0} u_{R} \rho_{12}\right)\left(\rho_{22}-\rho_{11}\right)-i \Delta_{\varepsilon} \rho_{12}+\xi_{0} u_{I}\left(\rho_{22}-\rho_{11}\right) \rho_{12}-\Gamma_{\varepsilon} \rho_{12} / 2, \\
\dot{\rho}_{21}=-i\left(\Omega_{0}+\xi_{0} u_{R} \rho_{21}\right)\left(\rho_{22}-\rho_{11}\right)+i \Delta_{\varepsilon} \rho_{21}+\xi_{0} u_{I}\left(\rho_{22}-\rho_{11}\right) \rho_{21}-\Gamma_{\varepsilon} \rho_{21} / 2, \\
\dot{\rho}_{22}=i \Omega_{0}\left(\rho_{12}-\rho_{21}\right)-2 \xi_{0} u_{I}\left|\rho_{12}\right|^{2}-\Gamma_{\varepsilon} \rho_{22},
\end{gathered}
$$

where $\Omega_{0}=g_{a} \varepsilon \cdot \sqrt{l_{R}^{2}+l_{I}^{2}}$ is the reduced Rabi frequency, $\Delta_{\varepsilon}=\Delta_{a}\left(l_{R}-\Gamma_{a}\left(n_{I} l_{R}+n_{R} l_{I}\right) /\left(2 \Delta_{a}\right)\right)$, $\Gamma_{\varepsilon}=\Gamma_{a}\left(n_{R} l_{R}-n_{I} l_{I}+2 \Delta_{a} l_{I} / \Gamma_{a}\right)$. Here $\Gamma_{a}$ is the relaxation rate from the excited state of the quantum dots, $\Delta_{a}$ is the frequency detuning of the probe field, $g_{a}=\mu_{a} \sqrt{\omega_{a} /\left(2 \hbar \varepsilon_{0} V\right)}$ is the coupling constant, $\varepsilon=A_{p}\left(\hbar \omega_{a} /\left(2 \varepsilon_{0} V\right)\right)^{-1 / 2}$, where $A_{p}$ is slowly varying amplitude of the probe field, $V$ is

\footnotetext{
${ }^{a}$ Corresponding author: avprokhorov33@mail.ru
} 
the quantization volume, $\rho_{a}$ is the density of quantum dots, $\omega_{a}=2 \pi c / \lambda_{a}$ is the frequency and $\mu_{a}$ is the dipole moment of the electronic transition. Because of the dispersive-dissipative properties of the host medium there appear the new terms in the equations (1), for which $u_{R}=\left(l_{R} \varepsilon_{R}+l_{I} \varepsilon_{I}\right) /\left(\varepsilon_{R}^{2}+\varepsilon_{I}^{2}\right)$, $u_{I}=\left(l_{I} \varepsilon_{R}-l_{R} \varepsilon_{I}\right) /\left(\varepsilon_{R}^{2}+\varepsilon_{I}^{2}\right)$, $l_{R}=\left(6\left(n_{R}^{2}+n_{I}^{2}\right)^{2}+3\left(n_{R}^{2}-n_{I}^{2}\right)\right) /\left(\left(2 n_{R}^{2}-2 n_{I}^{2}+1\right)^{2}+16 n_{R}^{2} n_{I}^{2}\right)$, $l_{I}=6 n_{R} n_{I} /\left(\left(2 n_{R}^{2}-2 n_{I}^{2}+1\right)^{2}+16 n_{R}^{2} n_{I}^{2}\right)$, taking into account the real $\varepsilon_{R}$ and imaginary $\varepsilon_{I}$ parts of the dielectric permittivity. If we choose the host medium to be an all-dielectric metamaterial it will allow us to change significantly the dispersion properties of the medium in the case $\varepsilon_{R}<0$ and $\mu<0$, where $\mu$ is the magnetic permeability of the medium. The presence of the dissipative corrections related to the local-field $\xi_{0} u_{I}\left(\rho_{22}-\rho_{11}\right) \rho_{21}$ in (1c) and $2 \xi_{0} u_{I}\left|\rho_{21}\right|^{2}$ in (1d) provides an additional possibility for the polarization decay of a-type quantum dots, which was induced by dissipative effects of the local field. Due to the nonlinear structure of these terms, it becomes possible to observe a variant of the self-induced transparency effect in the weak field limit for a dense ensemble of quantum dots. In this limit, the self-consistent problem described by (1) and propagation equation for $\mathbf{E}_{\mathbf{p}}$ can be reduced to a cubic-quintic Ginzburg-Landau equation [2]:

$$
\begin{aligned}
& \left(\frac{1}{v_{g}} \frac{\partial}{\partial t}+\frac{\partial}{\partial z}\right) \varepsilon+\frac{i}{2} \beta_{2} \frac{\partial^{2} \varepsilon}{\partial t^{2}}-i \frac{D_{0}}{2}\left(\frac{\partial^{2} \varepsilon}{\partial x^{2}}+\frac{\partial^{2} \varepsilon}{\partial y^{2}}\right)-i \gamma_{2}|\varepsilon|^{2} \varepsilon+i \gamma_{4}|\varepsilon|^{4} \varepsilon= \\
= & -\left(\alpha_{I}-i \alpha_{R}\right) \varepsilon-\alpha_{2}|\varepsilon|^{2} \varepsilon-\alpha_{4}|\varepsilon|^{4} \varepsilon+v_{2} \frac{\partial^{2} \varepsilon}{\partial t^{2}}+d_{x} \frac{\partial^{2} \varepsilon}{\partial x^{2}}+d_{y} \frac{\partial^{2} \varepsilon}{\partial y^{2}},
\end{aligned}
$$

where corresponding coefficients ( $\beta_{2}$ is the group velocity dispersion; $\gamma_{2}$ - cubic nonlinearity coefficient, $v_{2}$ is the spectral filtering; $\gamma_{4}$ is the fifth-order nonlinearity coefficient; $\alpha_{R}$ is the linear frequency modulation coefficient, $\alpha_{I}$ is the linear losses coefficient; $\alpha_{2}$ is the cubic nonlinear losses coefficient; $\alpha_{4}$ is the fifth-order losses coefficient) are functions of the material parameters presented in (1). Results of variational and numerical methods of searching stable dissipative laser bullets of the form $\varepsilon=A_{0} \exp \left[i\left(C_{0} X^{2}+D_{0} Y^{2}+F_{0} \tau^{2}+\Psi_{0}\right)-X^{2} / 2 X_{0}^{2}-Y^{2} / 2 Y_{0}^{2}-\tau^{2} / 2 \tau_{0}^{2}\right]$ are presented in fig. 1 .

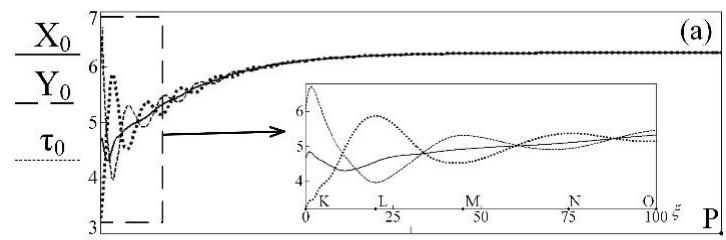

(a)

$\mathrm{A}_{0}$

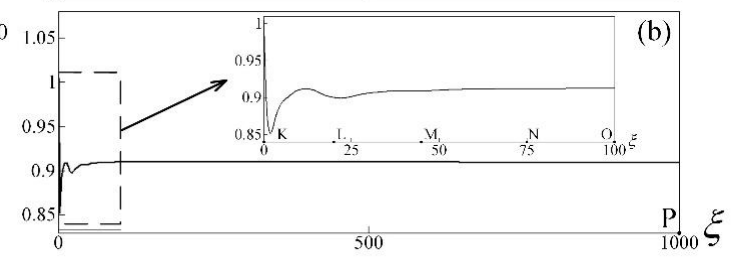

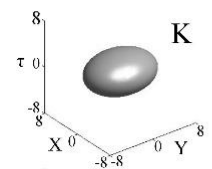

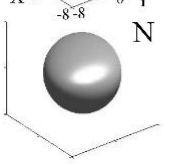

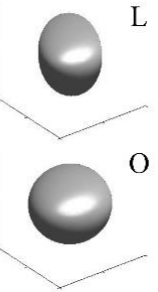

(c)

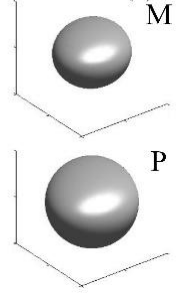

Figure 1. The process of stabilization (a) spatio-temporal $X_{0}, Y_{0}, \tau_{0}$ and (b) amplitude $A_{0}$ parameters of laser bullet, and (c) the transformation of its form in the medium of dielectric metamaterial with quantum dots $\mathrm{PbTe}$.

\section{References}

1. S.L. McCall, E.L. Hahn, Bull. Am. Phys. Soc. 10. P. 1189 (1965)

2. A.V. Prokhorov, M.G. Gladush, M.Yu. Gubin, et. al., Eur. Phys. J. D. 68:158 (2014) 